\title{
IMPACTO AMBIENTAL DERIVADO DAS AÇÕES DE CONTROLE E COMBATE A DENGUE NO RIO GRANDE DO NORTE
}

\author{
Carlos Enrique Jeronimo ${ }^{1}$, Lindemberg P. Nascimento ${ }^{2}$, Caio Pio Balbino ${ }^{3}$ \\ 1ㄷenrique@hotmail.com , ${ }^{2}$ nbergr@bol.com.br, ${ }^{3}$ caio_pio18@hotmail.com
}

\section{RESUMO}

A crescente presença dos problemas advindos da ocorrência do dengue no Brasil vem reforçando os elementos de controle aos vetores transmissores dessa doença. Entretanto, pouco se observa a aderência as questões ambientais associadas as técnicas utilizadas para o combate desses agentes, sobretudo, quando do uso de agentes químicos de grau de severidade elevado para a saúde humana. Sendo assim, o objetivo deste trabalho é alertar para uma realidade que esta fora da mídia e do conhecimento público, gerando elementos básicos para um processo de implantação de um sistema de gestão ambiental nas estruturas de controle de zoonoses. A metodologia utilizada para realização do trabalho foi revisão bibliográfica e aplicação da técnica FMEA para identificação e priorização dos principais aspectos e impactos ambientais. Os itens identificados foram priorizados em relação a sua ocorrência, freqüência, severidade, requisitos legais associados e medidas de controle utilizadas. Dos resultados obtidos é importante destacar o grau deste problema de saúde pública e ambiental, devendo ser estabelecido um ciclo de melhoria continua para apuração dos danos ambientais causados por tais atividades.

PALAVRAS-CHAVE: Dengue, inseticida, Impacto ambiental.

\section{ABSTRACT}

The growing presence of the problems arising from the occurrence of dengue in Brazil has been strengthening the control elements to vectors that transmit this disease. However, little is observed adherence environmental issues associated with the techniques used for combating these agents, especially when the use of chemical high severity to human health. Therefore, the objective is to draw attention to a reality that is out of the media and public knowledge, basic elements for generating a process of deploying an environmental management system in zoonoses control structures. The methodology used to conduct the study was a literature review and application of the FMEA technique for identifying and prioritizing key environmental aspects and impacts. The items identified were prioritized against its occurrence, frequency, severity, legal requirements and associated control measures used. From the results it is important to highlight the extent of the problem of environmental and public health, should be established a cycle of continuous improvement to calculate the environmental damage caused by such activities.

KEY-WORDS: Dengue, insecticide, environmental impact. 


\section{INTRODUÇÃO}

Nós últimos quinze anos o dengue tem sido a doença a estar presente nos 27 estados do Brasil, e que vem ganhando as maiores proporções enquanto impactos e são preocupantes os números de registros de óbitos, visto que centenas de pessoas no Brasil e cerca de 20 mil em todo o mundo vêm sendo vítimas fatais da referida doença, conforme descreve BRASIL (2010).

A Secretaria Estadual da Saúde Pública (SESAP), por meio do Programa Estadual de Controle da Dengue (PECD), do Estado do Rio Grande do Norte contabilizou 6.864 casos notificados de dengue, dos quais 1.164 foram confirmados. Atualmente, conforme descreve Diário de Natal (2012), 19 municípios registram alta incidência da doença, enquanto 23 encontra-se com média incidência e 84 municípios apresentam baixos índices. No entanto, 41 estão com incidência silenciosa e não se sabe se há subnotificação nesses locais. Sua acessão e associada e as condições climática e principalmente socioeconômicas.

A grande ameaça da expansão de doenças infecciosas como o dengue, conforme descrevem Chiaravalloti Neto F et al (2006), aponta para a necessidade da reestruturação da vigilância epidemiológica e da mudança das políticas de controle. Isto deve ter como base a visão de que a saúde pública é um problema amplo que não comporta soluções imediatistas, pois envolve a participação conjunta das agências governamentais e de toda a sociedade num processo contínuo.

Segundo Medronho (2008), o combate ao Aedes aegypti é o único elo passível de intervenção no controle do dengue, pois até o momento não existe vacina nem tratamento específico para a doença. No Brasil, as condições socioambientais e a ineficiência no combate ao vetor em muitas cidades contribuem sobremaneira para que o processo endêmico-epidêmico seja modulado pelo esgotamento de suscetíveis na população sob risco ao invés do controle do vetor.

Assim, as intervenções estão estritamente direcionadas para a eliminação do principal vetor desta enfermidade, o Aedes aegypti, que segundo Teixeira et al (2002), são realizadas mediante a execução de três linhas de ações, quais sejam: saneamento do meio ambiente, atividades de educação que visam a redução dos criadouros potencias deste mosquito, e o seu combate direto por meio de agentes químicos, físicos e biológicos. Nas áreas infestadas pelo Aedes aegypti, principalmente nas grandes metrópoles, estes programas demandam atividades intensas e contínuas durante todos os dias do ano, com visitas casa a casa e vultosos dispêndios de recursos.

Em geral, quando estas ações são desenvolvidas de acordo com as normas técnicas preconizadas, obtém-se expressiva redução da população de mosquitos, mensurada mediante redução dos índices de infestação (Índice Predial/IP, Índice de Breteau/IB e outros), conforme Mercadante (2006). Entretanto, tem-se observado que nem sempre esta redução é acompanhada de impacto sobre as infecções do vírus da dengue.

Entre as técnicas utilizadas pelas estruturas de gestão da saúde pública para o combate por agentes químicos são utilizados por meio da aspersão aeroespacial, (carro fumacê), técnica utilizada na eliminação do vetor que lança diretamente no meio ambiente inseticida. Entretanto, os impactos ambientais associados com tal técnica geram ações adversas, cujas alterações ecológicas e químicas devem ser controladas e monitoradas (Yang, 2003).

Contudo, as campanhas do combate a dengue, aparentemente, não possui escopo alinhadas nas práticas de prevenção à poluição, em especial pelo rigor nos aspectos ambientais associados às atividades, conforme preconizado na NBR-ISO 14001 (ISO, 2002). Necessitando do levantamento de uma matriz abrangente, com o tratamento dos aspectos e impactos ambientais 
associados a tais atividades.

Sendo assim, o objetivo deste trabalho consiste no levantamento e priorização dos aspectos e impactos ambientais, mediante a utilização de uma metodologia de levantamento matricial das atividades que envolvem as ações de prevenção e controle de vetores do dengue. Além disso, espera-se que tal descrição (visto a escassez na literatura) sirva de alerta para a gravidade do impacto ambiental negativo gerado e associado ao combate a dengue ao meio ambiente. Bem como, os desdobramentos ocasionados enquanto influência em fenômenos como: alterações climáticas, aquecimento global, danos a biodiversidade e poluição de recursos hídricos.

\section{METODOLOGIA}

O estudo constitui-se de uma pesquisa aplicada, pois objetiva levar conhecimentos para extensão das tecnologias e impactos advindos das atividades relativas ao controle de vetores do dengue, levantadas e avaliadas em levantamento técnico e bibliográfico.

Do ponto de vista dos objetivos, trata-se de um estudo exploratório-descritivo, o qual visa descrever a problemática em discussão, buscando caracterizar o objeto de estudo.

A hipótese pauta-se na observância de impactos as matrizes ar, água e solo; por ações derivadas do controle de vetores ambientais relacionados a proliferação do dengue.

Do ponto de vista dos procedimentos técnicos, o estudo caracteriza-se como pesquisa bibliográfica, por sua elaboração partir do levantamento e análise de material já publicado, em artigos científicos, livros, relatórios técnicos, etc. (SILVA; MENEZES, 2001), e como estudo de caso devido à utilização de dados de campo, com observação assistemática não-participante, in loco.

A estrutura da pesquisa consiste em:

- Formulação do problema, englobando a justificativa do estudo, a determinação dos objetivos, a contextualização da problemática e definição da metodologia;

- Realização do levantamento teórico, que orienta a caracterização do objeto de estudo, as definições e conceitos a serem utilizados em análise e correntes de pensamentos que norteiam a hipótese da pesquisa;

- Levantamento de dados em campo, por meio de incursões investigativas em zonas de riscos no estado do Rio Grande do Norte;

- Estudo criterioso sobre os princípios do combate aos vetores e as ações a serem aplicadas na prática das atividades apresentadas, que tenham viabilidade técnica;

- Apresentação das oportunidades de aplicação identificadas e discussão sobre as melhorias que possam acarretar.

Os dados coletados em campos foram organizados, de acordo com a necessidade da utilização em pesquisa, e utilizados para elaboração do levantamento das principais ações de oportunidades.

\section{Área de Estudo}

O desenvolvimento do estudo foi realizado em zonas periféricas do município de Natal, com acompanhamento das atividades realizadas pelos profissionais do controle de zoonoses do 
município, na busca de mapear o processo e desenvolvimento dos principais impactos ao meio ambiente.

\section{Levantamento dos Aspectos e Impactos ao Meio Ambiente}

De acordo com a norma de orientação da NBR-ISO 14001:1996, o relacionamento entre aspectos ambientais e impactos ambientais é o de causa e efeito. $O$ aspecto ambiental é a causa, como a emissão de algo no ar; o efeito é o impacto no meio ambiente, como, por exemplo, o aumento do nível de emissão no ambiente da fábrica. O levantamento dos dados foi realizado seguindo o modelo de Moreira (2000), adaptado por Campani et al. (2005), o qual se baseia na utilização da ferramenta FMEA, o que possibilita identificar e ordenar os impactos ambientais de maiores prioridades.

Sendo assim, seguindo a orientação da norma foi adotado um procedimento de quatro etapas para identificar os aspectos e impactos associados as atividades de prevenção e combate ao dengue, a saber:

a) Seleção das atividades ou processos (por exemplo, manuseio de materiais prejudiciais);

b) Identificação de todos os aspectos ambientais possíveis das atividades ou processos (por exemplo: derramamentos acidentais em potenciais);

c) Identificação dos impactos reais ou potenciais associados com o aspecto (por exemplo: nível de contaminação do solo e/ou água); e

d) Avaliação da importância dos impactos, seguindo critério de pontuação padrão.

Uma vez definidos os impactos ambientais, é necessário determinar sua importância. Para avaliá-la, adotaram-se o critério sugerido pela norma de orientação ISO-14004 (ISO, 2002), tendose como fatores os seguintes itens: escala do impacto; sua gravidade; a probabilidade de sua ocorrência e a duração do impacto.

Esta forma de avaliação permitiu identificar os aspectos ambientais das atividades de prevenção e combate ao dengue, em especial a partir do seu fluxograma de processo, determinando-se os impactos ambientais associados a estes aspectos e avaliar sua importância. Como registro desta avaliação, utilizou-se a Figura 1, cuja representação consiste na Planilha de Avaliação de Aspectos Ambientais. Essa metodologia foi baseada em Novato et al (2006).

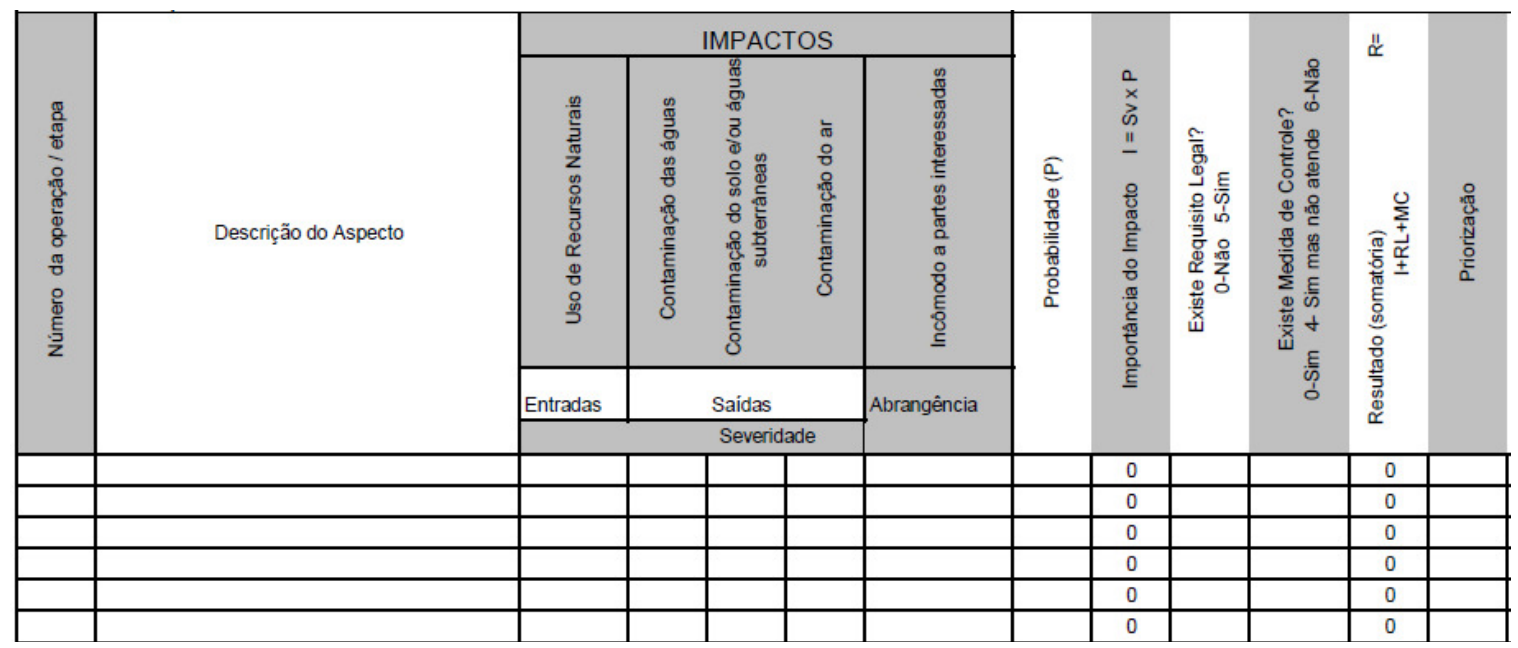

Figura 1. Planilha para registro dos aspectos, impactos e grau de priorização. 
O ciclo de análise consistiu com o levantamento de todos os elementos de entrada e saída do macrofluxo de combate ao dengue, estabelecendo a partir deste, o marco da análise. $\mathrm{Na}$ Tabela 1, é apresentado o primeiro passo, relativo ao consumo dos agentes e recursos naturais. Tais elementos somados aos graus de abrangência e alcance das conseqüências destes aspectos formulam pelo somatório o grau de severidade do impacto. Ou seja, nas Tabelas 2 e 3 , são apresentados os elementos complementares e na Equação 1, o modelo de cálculo utilizado.

Tabela 1. Grau de Severidade para os Aspectos Associados.

\begin{tabular}{|c|c|c|}
\hline $\begin{array}{c}\text { Matérias Primas e } \\
\text { Insumos (MP) } \\
\text { (Consumo/mês) }\end{array}$ & Severidade (Produtos Perigosos) & Severidade (Prod. Não Perigosos) \\
\hline 25-30\% do consumo total & $\mathbf{2}$ & $\mathbf{1}$ \\
\hline 30-60\% do consumo total & $\mathbf{3}$ & $\mathbf{2}$ \\
\hline $\begin{array}{c}61-100 \% \text { do consumo } \\
\text { total }\end{array}$ & $\mathbf{4}$ & $\mathbf{3}$ \\
\hline
\end{tabular}

Tabela 2. Grau de Abrangência dos Impactos Ambientais Associados

\begin{tabular}{|c|c|c|}
\hline Nível de Abrangência (A) & Descrição & Peso \\
\hline Baixa & $\begin{array}{c}\text { Eventos que afetam o meio ambiente, } \\
\text { mas que por meio de ação simples e } \\
\text { imediata, o potencial dano pode ser } \\
\text { remediado. }\end{array}$ & $\mathbf{1}$ \\
\hline Média & $\begin{array}{c}\text { Eventos que atingem o meio } \\
\text { ambiente, mas que por meio de ação } \\
\text { simples e imediata, com a } \\
\text { disponibilização de recursos e/ou } \\
\text { apoio remedia o potencial dano. }\end{array}$ & $\mathbf{2}$ \\
\hline Alta & $\begin{array}{c}\text { Eventos que tem a potencialidade de } \\
\text { causar danos significativos ao meio } \\
\text { ambiente. }\end{array}$ & $\mathbf{3}$ \\
\hline
\end{tabular}

Tabela 3. Nível de Abrangência dos Impactos Analisados

\begin{tabular}{|c|c|c|}
\hline Nível de Alcance (AA) & Descrição & Peso \\
\hline Local & $\begin{array}{c}\text { Restrito aos limites do setor de } \\
\text { trabalho }\end{array}$ & $\mathbf{1}$ \\
\hline Regional & Restrito aos limites da empresa & $\mathbf{3}$ \\
\hline Global & $\begin{array}{c}\text { Atinge áreas além dos limites da } \\
\text { organização, toda a comunidade. }\end{array}$ & $\mathbf{5}$ \\
\hline
\end{tabular}

Severidade $(S)=M P+A+A A$

(Eq. 1)

Seguindo o fluxo da análise são avaliados os graus de probabilidade destes aspectos, que compõe o grau de intensidade destes. Na Tabela 4 apresenta-se o critério do nível de probabilidade e na Equação 2 o modelo de cálculo da intensidade. 
Tabela 4. Probabilidade dos Impactos Analisados

\begin{tabular}{|c|c|c|}
\hline Probabilidade $(\mathrm{P})$ & Descrição & Peso \\
\hline Baixo & O aspecto ocorre esporadicamente. & $\mathbf{1}$ \\
\hline Médio & O aspecto ocorre frequentemente. & $\mathbf{3}$ \\
\hline Alto & $\begin{array}{c}\text { O aspecto ocorre continuamente, } \\
\text { ininterruptamente. }\end{array}$ & $\mathbf{5}$ \\
\hline
\end{tabular}

Intensidade $(I)=$ Severidade $(S) x$ Probabilidade $(P)$

(Eq. 2)

Por fim, são analisados os elementos do cumprimento aos requisitos legais associados (respeitando os impactos associados), por meio dos critérios da Tabela 5. E analisado as medidas de controle dos impactos advindos, por meio dos elementos da Tabela 6. Logo, obtém-se pela Equação 3 o grau do impacto da atividade associado, ou melhor, seu aspecto ambiental.

Tabela 5. Atendimento aos Requisitos Legais Associados aos Aspectos Analisados

\begin{tabular}{|c|c|c|}
\hline Atendimento Legal (RL) & Descrição & Peso \\
\hline Eficaz & Eficaz, atende a legislação & $\mathbf{0}$ \\
\hline Eficiente & Eficiente, mas não atende a legislação & $\mathbf{4}$ \\
\hline Ineficiente/Ineficaz & Não é eficiente e não atende a legislação & $\mathbf{6}$ \\
\hline
\end{tabular}

Tabela 6. Medidas de Controle para Prevenção dos Impactos Analisados

\begin{tabular}{|c|c|c|}
\hline Medida de Controle (MC) & Descrição & Peso \\
\hline Eficaz & Eficaz, atende a legislação & $\mathbf{0}$ \\
\hline Eficiente & Eficiente, mas não atende a legislação & $\mathbf{4}$ \\
\hline Ineficiente/Ineficaz & Não é eficiente e não atende a legislação & $\mathbf{6}$ \\
\hline
\end{tabular}

$\operatorname{Re} \operatorname{sultado}(R)=$ Intensidade $(I)+\operatorname{Re}$ quisitos Legais $(R L)+$ Medidas de Controle $(M C)$

(Eq. 3)

\section{RESULTADOS E DISCUSSÕES}

Diante do levantamento dos aspectos ambientais associados a atividade foi elencado o cenário apresentado na Tabela 7, onde tem-se uma composição de 20 impactos aos meio ambiente, advindos da referida atividade. 
Tabela 7. Aspectos e Impactos Ambientais Levantados

\begin{tabular}{|l|l|l|}
\hline Atividade & Aspectos & Impactos \\
\hline Varredura e Inspeçães & $\begin{array}{l}\text { Consumo de Combustíveis Fósseis e e } \\
\text { Consumo de Papel }\end{array}$ & $\begin{array}{l}\text { Poluição do ar (1) e Esgotamento de } \\
\text { Recursos Naturais (2) }\end{array}$ \\
\hline Combate Químico Direto & $\begin{array}{l}\text { Consumo de Produto Químico, Geração } \\
\text { de Efluentes e Geração de Vapores } \\
\text { Orgânicos }\end{array}$ & $\begin{array}{l}\text { Esgotamento de recursos naturais (3), } \\
\text { Contaminação do Solo (4), Contaminação } \\
\text { da Água (5), Mortandade de Espécies (6) e } \\
\text { Doenças pela exposição a agentes químicos } \\
\text { (7). }\end{array}$ \\
\hline $\begin{array}{l}\text { Aspersão de Agentes } \\
\text { Químicos }\end{array}$ & $\begin{array}{l}\text { Consumo de Produtos Químicos e e } \\
\text { Combustíveis, Geração de Efluentes e e } \\
\text { Geração de Vapores Orgânicos }\end{array}$ & $\begin{array}{l}\text { lsgotamento de recursos naturais (8), } \\
\text { Contaminação do Solo (9), Contaminação } \\
\text { da Água (10), Mortandade de Espécies (11) } \\
\text { e Poluição do Ar (12). }\end{array}$ \\
\hline Remoção de Resíduos & Geração de Resíduos Sólidos & $\begin{array}{l}\text { Contaminação do solo (13) e Deslocamento } \\
\text { de Passivo Ambiental (14) }\end{array}$ \\
\hline Processo Educativo & Consumo de Papel & $\begin{array}{l}\text { Geração de resíduos sólidos (15) e } \\
\text { Esgotamento de Recursos Naturais (16) }\end{array}$ \\
\hline $\begin{array}{l}\text { Sução de Efluentes de } \\
\text { Grande Concentração de } \\
\text { Focos }\end{array}$ & $\begin{array}{l}\text { Geração de Efluentes e Consumo de de } \\
\text { Combustíveis }\end{array}$ & $\begin{array}{l}\text { Poluição da água (17), Poluição do Solo } \\
\text { (18), Poluição do ar (19) e Esgotamento de } \\
\text { recursos Naturais (20). }\end{array}$ \\
\hline
\end{tabular}

Para a priorização e identificação do grau de risco associado, foi aplicada a metodologia referenciada no item especifico, com aplicação da planilha de critérios. Os resultados obtidos são apresentados na Figura 2. A escala de priorização consiste em: vermelho - críticos, amarelo - significativo, azul - alerta, verde - monitoráveis e branco - negligenciáveis.

Dos resultados obtidos observa-se uma grande intensidade de pontos na faixa branca, que consistem nos impactos de pequeno grau de significância, não sendo importantes para priorização na gestão da atividade. Entretanto, os demais pontos estão concentrados na faixa que requer o monitoramento continuado e níveis de alerta. Logo, elementos mitigadores devem ser utilizados para: evitar a contaminação do solo, da água e do ar. Tais resultados seguem mesmo padrão identificado em estudo semelhante realizado por Novato et al (2006).

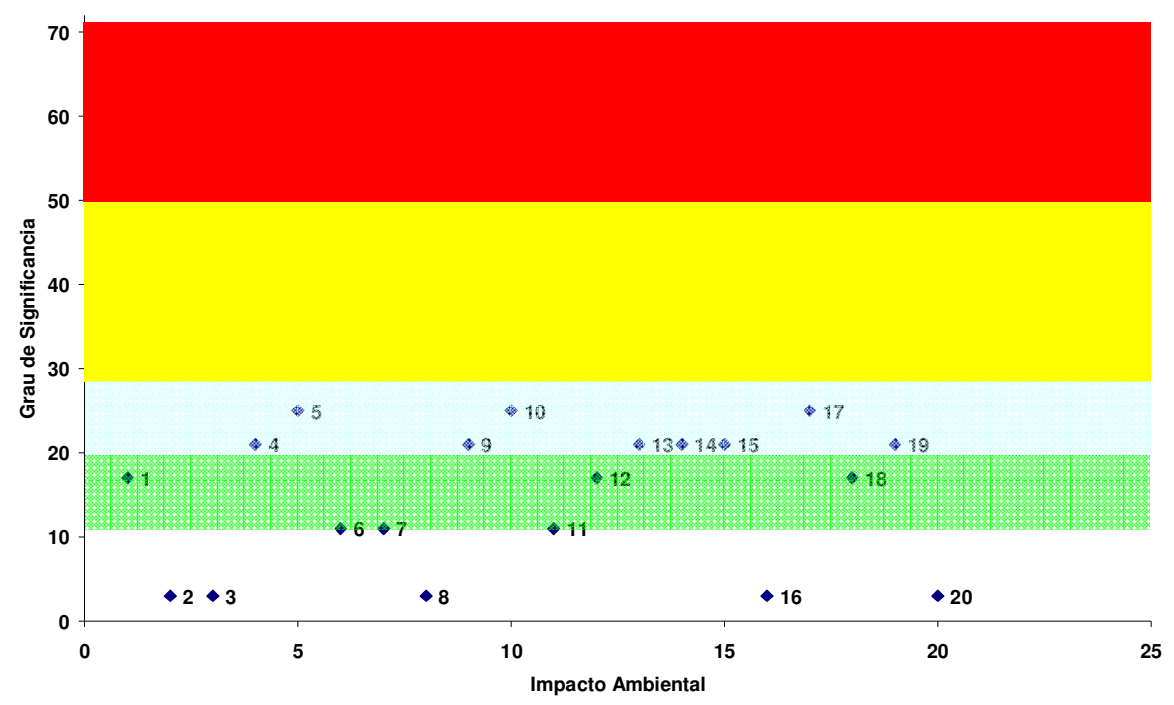

Figura 2. Dispersão do grau de criticidade dos impactos analisados. 
Pela analise o consumo de recursos naturais na atividade é considerado desprezível, não estando entre os elementos principais para ações de gestão e estratégias de mitigação da sua ocorrência.

Um parêntese deve ser aberto para algumas modificações do cenário de combate ao dengue, que vem ocorrendo em conseqüência do aquecimento global. Visto que os insetos vetoriais são sensíveis as condições meteorológicas a elevação da temperatura ocasiona uma reprodução e amadurecimento mais rápido dos seus agentes patogênicos. Os mosquitos proliferam mais rápidos e picam mais, quando o ar é mais quente (Souza, 1999).

Ao mesmo tempo, o calor mais intenso acelera a taxa em que os agentes patogênicos se reproduzem e amadurecem. Logo, é necessário admitir que a mudança climática, provavelmente, venha a ter efeito indiretos sobre as populações vetoras. Assim sendo, em decorrência das alterações ambientas uma delas poderá ser substituída por outra. Deste que a capacidade vetora de ambas poderia divergir, a transmissão dependerá das características das espécies envolvidas. Assim, se um desses vetores desaparece como ocorre em conseqüência de desmatamento, poderá dar-se a eliminação do problema ou então a continuidade ou mesmo o incremento epidemiológico da doença (Forattini, 2004).

Outro ponto importante, citado por Donalísio; Glasser (2002) que afirmam que além dos vários mecanismos de resistência presentes nos insetos, que permitem sua sobrevivência após contato com o inseticida, há outra forma, chamada de comportamental, que define o processo de seleção de indivíduos com aptidão para evitar total ou parcialmente o contato com doses que resultariam letais. O fumacê é um veneno em forma de óleo, transformado em gotículas por uma máquina especial que o espalha pelas ruas. Esse veneno cobre todas as superfícies, como telhados, plantas, janelas e gramados. Em um dia de chuva, o material é "varrido" destes locais e cai nos leitos d'água (rios e lagos, principalmente). Tem-se então o envenenamento destes locais, que resulta na morte de micróbios e pequenos insetos, prejudicando o equilíbrio ambiental deste sistema. "Os peixes, que dependem de animais menores para se alimentar, também morrerão". Partindo para o ambiente terrestre, os pássaros sofrerão um grande impacto negativo, pois os seres vivos de que se alimentam como besouros e formigas, não existirão em quantidade suficiente. Então, pouco se sabe sobre esse veneno e seus efeitos a médio e longo prazo. Esse produto, muitos acreditam que seu uso seria a melhor solução para o combate a Dengue. Já existe teste em laboratório que demonstraram que a maior parte dos mosquitos não morre mais com o fumacê.

Portanto, o modelo aqui descrito não deve ser considerado como um elemento estático, visto que pelos motivos citados acima se tem um cenário bastante mutável.

Em relação aos impactos associados a tal atividade elencou-se um quadro de possíveis medidas mitigadoras que devem ser adotadas para minimização dessas conseqüências ambientais, a saber:

- Estabelecer indicadores de monitoração da qualidade do ar, solo e água em áreas cuja freqüência de atividades seja recorrente;

- Fortalecer campanhas educativas para mitigação das causas que eliminariam o combate aos vetores;

- Estabelecer mecanismos que estabeleçam a certificação dos organismos de controle de zoonoses em aderência a sistemas de gestão conforme NBR-ISO 14001;

- Estabelecer um plano de gerenciamento de resíduos sólidos, com destinações adequadas para os resíduos provenientes da atividade. 


\section{CONCLUSÕES}

O estudo permitiu o levantamento dos principais aspectos ambientais, e respectivos impactos ao meio ambiente, por meio da obtenção de uma matriz de priorização, considerando elementos de abrangência, alcance, severidade, probabilidade, requisitos legais associados e medidas de controle integradas ao processo.

Foram descritos vinte impactos associados a atividade, onde nenhum destes foram enquadrados em níveis emergenciais ou críticos de controle. Entretanto, foi observada uma ocorrência significativa de elementos nas classes que requerem monitoração e medidas imediatas para mitigação.

O estudo também define que tal cenário é mutável, necessitando de freqüentes adaptações e ciclo contínuo de melhoria, daí a recomendação para que as estruturas de controle de zoonoses implementem sistemas de gestão ambiental baseados nos critérios da NBR-ISO 14001.

\section{REFERÊNCIAS}

ANDRADE, M. R. S.; TURRIONE, J. B. Uma metodologia de análise dos aspectos e impactos ambientais através da utilização do FMEA. In: Encontro Nacional de Engenharia de Produção/ ENEGEP, São Paulo: 2000.

APERJ, Fundação Carlos Chagas Filho de Amparo à Pesquisa do Estado do Rio de Janeiro: Dengue põe em evidência pesquisas realizada no Rio de Janeiro. Disponível em:

http://www.faperj.br/boletim_interna.phtml?obj_id=4424. Acesso: em 10 do abr.2012.

BRASIL, Ministério da Saúde. Secretaria de Vigilância em Saúde. Departamento de Vigilância

Epidemiológica. Doenças infecciosas e parasitárias: Ministério da Saúde: 8.ed. Brasília. Ministério da Saúde. 2010.

CAMPANI, D. B. et al. A Gestão Ambiental da Universidade Federal do Rio Grande do Sul. In: V Congreso de AIDIS-sección uruguaya, 2005.

CHIARAVALLOTI, F. et al. Controle do dengue em uma área urbana do Brasil: avaliação do impacto do Programa Saúde da Família com relação ao programa tradicional de controle. Cad. Saúde Pública, Rio de Janeiro, 22(5): 987-997, mai, 2006.

DIARIO DE NATAL. Disponível em: http://www.donline.com.br. Acesso em: 20/06/2012.

DIGGLE, P. J. (1992). Time Series. A Biostatistical Introduction. In (Copas, J.B. et al., eds.): Oxford Statistical Science Series - 5. Oxford Science Publications. Oxford

DONALÍSIO, M. R.; GLASSER, C. M. Vigilância entomológica e controle de vetores do dengue. Rev.Bras. Epidemiologia, v. 5, n. 3, p. 259-272, 2002.

FORATTINI, O. P. Ecologia epidemiologia e socidade.-2.ed.-São Paulo :Artes Médicas, p.35, 2004.

ISO. International Organization for Standardization. Environmental management - the ISO 14000 family of international standards. 2002.

MEDRONHO, R. A. Dengue no Brasil: desafios para o seu controle. Cad. Saúde Pública, Rio de Janeiro, 24(5): 948-949, mai, 2008.

MERCADANTE Jr, E. Dengue ameaça o Rio.Rio de Janeiro: Conselho Regional de Biologia 2a. região RJ/ES. BIONOTÍCIAS, ano $x, n^{\circ}$ 7, jan/fev, p.5, 2006.

MONTEIRO, C. A. (Org) - Velhos e Novos males da saúde no Brasil: A evolução do país e suas doenças. São Paulo: HUCITEC, NUPENS/USP, - 2a ed., 2000. 
MOREIRA, M. S. Estratégia e Implantação do Sistema de Gestão Ambiental (Modelo ISO1400). Editora DG. NBR ISO 14001: Sistemas de Gestão Ambiental: especificações e diretrizes para uso. ABNT, Rio de Janeiro, 1996.

NOVATO, A. P. S. et al. Levantamento e priorização de oportunidades de produção mais limpa para os recursos hídricos em refinarias de petróleo. Trabalho final de curso, especialização em processamento de petróleo. Instituto de Química, UERJ/Petrobras, Rio de Janeiro, 2006.

RUEDA, et al. (1990). Temperature Development and Survival Rates of Culex quinquefasciatus and Aedes aegypti (Dipetera: Culicidae). Journal of Medical Entomology 27 (5): 892 -898.

SILVA, E. L.; MENEZES, E. M. Metodologia da pesquisa e elaboração da dissertação. 30 ed. Universidade Federal de Santa Catarina - UFSC: Florianópolis, 2001.

SANTOS, R. S. Fatores associados à ocorrência de formas imaturas de Aedes aegypti na llha do Governador, Rio de Janeiro, Brasil. Uberaba: Revista da Sociedade Brasileira de Medicina Tropical, vol.32, n. ${ }^{\circ} 4$, Jul/Ago, 1999.

TEIXEIRA, M. G. et al. Avaliação de impacto de ações de combate ao Aedes aegypti na cidade de Salvador, Bahia. Rev. Bras. Epidemiol. Vol. 5, № 1, 2002.

YANG, H. M et al. The Serorevertion and The Survival Related to HIV Infection Among Children: Statistical Modeling Applied to Retrospective Data Collection. Mathematical and Computer Modelling: Estados Unidos: ,v.38, p.251 - 267, 2003. 